\title{
Ingeniería de Procesos Aplicada a la Obtención de Bioetanol Usando el Rechazo de Banano de LA ZoNa dE URABá
}

\author{
Process Engineering Applied to the Production \\ of Bioethanol Using Banana Rejection Urabá
}

\author{
Flórez Alvarado Daniel'1, Lara Sandoval Adriana² \\ ${ }^{1}$ Corporación para el Desarrollo Sostenible del Uraba - CORPOURABA, \\ Antioquia, Colombia. urikox@hotmail.com \\ 2 Universidad Nacional Abierta y a Distancia UNAD, Escuela de Ciencias Básicas Tecnología e Ingeniería, \\ Boyacá, Colombia. Adrianalara_2@hotmail.com
}

Recibido: 17/10/2011 • Aceptado: 15/12/2011

\section{RESUMEN}

La mayoría de los países ya sea económicamente avanzados o en diferentes etapas de desarrollo se enfrentan con el problema de la eliminación y el tratamiento de los desechos y residuos orgánicos; estos pueden tratarse de diferentes maneras; por ejemplo, mediante la reducción de su volumen o por procesamiento en alguna sustancia útil utilizando los procesos fisicoquímicos de transformación del banano en bioetanol y analizando los impactos ambientales para cumplir con las normas sanitarias.

El objetivo de este trabajo se centró en evaluar el potencial de producción de etanol a partir de banano y el estudio y aplicación de la ingeniería de procesos en la obtención de etanol utilizando banano de rechazo como materia prima en la región de Urabá.

El bioetanol se obtuvo a través de la fermentación y destilación del banano de rechazo, cuyos resultados se plasman en los controles operacionales y en la hoja patrón que se obtuvo de la estandarización del proceso.

Palabras clave: banano, bioetanol, destilación, fermentación, mosto

\section{ABSTRACT}

Most countries either financially or in advanced stages of development are faced with the problem of disposal and treatment of waste and organic waste; these can be treated in different ways, for example by reducing its volume or processing any useful substance using physicochemical transformation processes bananas in bioethanol and analyzing environmental impacts to meet sanitary standards. 
The objective of this study was to evaluate the potential for ethanol production from banana and the study and application of process engineering in ethanol production using banana rejection as feedstock in the region of Urabá.

Bioethanol is obtained by fermentation and distillation of rejected bananas where the results are reflected in the operational controls and leaf pattern obtained in the standardization process.

Keywords: bananas, bioethanol, distillation, fermentation, must

\section{INTRODUCCIÓN}

La producción de etanol a partir de cereales y caña de azúcar carece de soluciones económicas competitivas y ha contribuido al incremento del costo de los alimentos. Por otro lado, según [1] en el mundo se producen aproximadamente 1.600 millones de toneladas por año de residuos sólidos [2]. "En el caso de Colombia, el $65 \%$ son residuos orgánicos entre los cuales se encuentran los de subproductos y de rechazo agrícolas"[3].

"La producción de banano de exportación (variedad Cavendish valery) desató un problema ambiental, dadas las exigencias en el control de calidad que acarrearon rechazos de fruta entre $20 \%$ y $25 \%$ v/v" [4].

El banano es una materia prima apta para la obtención de bioetanol. Presenta un alto contenido de carbohidratos (aproximadamente $20 \%$ de su peso) idóneos para procesos fermentativos encaminados a la producción de alcohol [5]. "La producción de bioetanol se realiza mediante un proceso fermentativo en el cual se utilizan microorganismos capaces de convertir el azúcar en etanol. Usando levaduras S. cerevisiae para la producción de etanol en fermentaciones tradicionales en batch, la productividad está limitada entre 1.8 a $2.3 \mathrm{~g} / \mathrm{Lh} . "$ [5].

"Las estrategias para la retención celular incluyen la separación en la corriente de producto, seguida de un reciclo al fermentador o inmovilización dentro del fermentador." [5]. Esta última técnica ha sido previamente estudiada debido a los beneficios que presenta, comparada con los procesos con células libres, ya que ayuda a mantener la actividad enzimática garantizando una buena actividad celular durante la fermentación, de tal modo que permite una reducción de los tiempos de proceso, entre otras ventajas [4].

"En las últimas décadas, la utilización de procesos biotecnológicos para la obtención de bienes y servicios se ha convertido en una gran alternativa que ofrece soluciones a problemas provenientes de la aplicación de tecnologías tradicionales. En Colombia, se están obteniendo avances tecnológicos y productivos importantes en la aplicación de la biotecnología en cuanto a la producción de biocombustibles se refiere" [5].

Urabá goza de condiciones climáticas excepcionales para la producción del banano, puesto que la riqueza de su suelo ha permitido que la región se convierta en un productor agrícola de excelente calidad, ya que esta fruta se encuentra disponible todo el año.

"Muchos bananeros están implementando sistemas de compostaje de este banano de desecho para devolverlo a las fincas en forma de abono orgánico. El concepto es bueno. El gremio necesita, sin embargo, mejorar estas plantas de "compostaje", lo que se puede conseguir optimizando el sistema tecnológico [6]. "La producción de etanol, a partir del banano rechazado, es una tendencia ecológica a frenar la contaminación producida 
por los combustibles fósiles por utilización de este alcohol. Esta producción se hará sin desabastecer el ámbito alimenticio y de comercialización interna del banano" [7].

\section{Marco Teórico}

\section{A. Etanol}

El etanol, o alcohol etílico, es una sustancia con formula molecular $\mathrm{C}_{2} \mathrm{H}_{6} \mathrm{O}$, que puede ser utilizada como combustible en motores de combustión interna con ignición a chispa de dos maneras, básicamente: en mezclas de gasolina y etanol anhidro; o como etanol puro, generalmente hidratado [8].

\section{B. Biomasa}

Es toda sustancia orgánica renovable de origen tanto animal como vegetal; la energía de la biomasa proviene de la energía que almacenan los seres vivos. En primer lugar, los vegetales realizan la fotosíntesis para lo cual utilizan la energía del sol y, de esta manera, formar sustancias orgánicas. Después, los animales incorporan y transforman esa energía al alimentarse de las plantas. Los productos de dicha transformación que se consideran residuos, pueden utilizarse como recurso energético" [9]. La biomasa no emite contaminantes sulfurados o nitrogenados, ni partículas sólidas; por el contrario, si se utilizan residuos de otras actividades como biomasa esto se traduce en un reciclaje y disminución de residuos [10].

\section{Biocombustibles}

Son aquellos combustibles que se producen a partir de la biomasa y que se consideran, por tanto, energía renovable. Se pueden presentar en forma sólida (residuos vegetales, fracción biodegradable de los residuos urbanos o industriales), líquida (bioalcoholes, biodiésel) y gaseosa (biogás, hidrógeno). Dentro de los biocombustibles, los biocarburantes abarcan al subgrupo caracterizado por la posibilidad de su aplicación a los actuales motores de combustión interna (motores diésel y Otto). Son, en general, de naturaleza líquida [11].
Los biocarburantes en uso proceden de materias primas vegetales, a través de transformaciones biológicas y físico-químicas. Actualmente, se encuentran desarrollados principalmente dos tipos: el biodiésel, obtenido a partir de la transesterificación de aceites vegetales y grasas animales con un alcohol ligero, como metanol o etanol; y el bioetanol, obtenido fundamentalmente de semillas ricas en azúcares mediante fermentación[12].

\section{Bioetanol}

Se obtiene de forma natural por la fermentación de azúcares y almidones de múltiples plantas. Biocarburante, combustible de origen vegetal que tiene características parecidas a las de los combustibles fósiles, lo que permite su utilización en motores apenas modificados; además, no contiene azufre, uno de los principales causantes de la lluvia ácida. Se obtiene por fermentación de medios azucarados extraídos a partir de caña de azúcar, remolacha, maíz, trigo, banano y cebada, entre otros. Se utiliza para incrementar el octanaje y mejorar la calidad de las emisiones de la gasolina, al convertirla en un combustible oxigenado [13].

El bioetanol, como combustible de transporte, se utiliza de diferentes formas; por ejemplo, mezclado con gasolina en bajos porcentajes (menores de $5-10 \%$ ), los alcoholes aumentan el contenido de oxígeno de esta y con ello su octanaje; así arden mejor y optimizan el funcionamiento del vehículo sin que haya que modificar los motores, al mismo tiempo que reducen el consumo y las emisiones contaminantes [14].

\section{E. Subproductos de la obtención del bioetanol}

"Los subproductos generados en la producción de bioetanol, así como el volumen de los mismos, dependen en parte de la materia prima utilizada. En general se pueden agrupar en dos tipos" [15].

1) Materiales lignocelúlicos: "Tallos, bagazo, etc., correspondientes a las partes estructurales de la planta. En general, se utilizan para valorización energética en cogeneración, especialmente 
para cubrir las necesidades energéticas de la fase de destilación del bioetanol, aunque también se puede vender el excedente a la red eléctrica (con precio primado)" [15].

2) Materiales alimenticios: pulpa y granos de destilería desecados con solubles (DDGS), que son los restos energéticos de la planta después de la fermentación y destilación del bioetanol; tienen interés para el mercado de piensos animales por su riqueza en proteína y valor energético [10].

\section{F. Banano}

Pertenece a la familia de las Musáceas, tiene su origen en Asia meridional y se conoce en el Mediterráneo desde el año 650 d.C.; es el cuarto cultivo de frutas más importante del mundo, su peso oscila en torno a los 100-120 gramos, su piel puede ser de color amarillo verdoso, amarillo y amarillo-rojizo. Existe un gran número de variedades de cultivo en Oriente; cada región tiene sus propias variedades adaptadas a las condiciones climáticas locales; sin embargo, las variedades introducidas en los trópicos americanos son mucho más limitadas [13].

\section{G. Hidrólisis}

Las celulosas no pueden ser fermentadas directamente; es necesario convertirlas en azúcares más sencillos para su transformación en alcohol. La hidrólisis es un proceso químico que divide la molécula de celulosa por la acción de la molécula de agua. Las complejas estructuras de la celulosa (celulosa, hemicelulosa y lignina) se dividen en diferentes procesos para conseguir una solución azucarada, y eliminar productos de descomposición de los azúcares que pueden inhibir o, al menos, dificultar el proceso de fermentación. Principalmente, se realizan procesos de hidrólisis de ácidos concentrados y bajas temperaturas, de ácidos diluidos y altas temperaturas y enzimáticos [16].

\section{H. Fermentación}

La fermentación alcohólica es la transformación de sustratos azucarados por la acción de microorganismos, que durante su ciclo de vida consumen el sustrato y fabrican etanol y otros compuestos bioquímicos, como resultado de su metabolismo [8].

\section{Destilación}

El alcohol producido por destilación contiene una parte significativa de agua, que debe ser eliminada para su uso como combustible. Para ello, se utiliza un proceso de destilación. Dado que el etanol tiene un punto de ebullición menor $\left(78,3^{\circ} \mathrm{C}\right)$ que el agua $\left(100^{\circ} \mathrm{C}\right)$, la mezcla se calienta hasta que el alcohol se evapore y se pueda separar por condensación de este [17].

\section{J. Impacto ambiental}

Entre los impactos que produce en el ambiente se incluyen los efectos contrarios producidos por los cultivos intensivos, la polución debida a los procesos de fermentación y otros. Muchos de estos efectos se pueden disminuir mediante un diseño más sensible, una localización apropiada y la correspondiente investigación en medida y precaución de la polución.

Con el $10 \%$ en volumen de etanol en la gasolina, se reducirá hasta 1.6 millones de toneladas de $\mathrm{CO}_{2}$ por año para una producción de $300 \mathrm{KI}$ por día de etanol [18].

\section{Metodología de LA INVESTIGACIÓN}

\section{A. Tipo de investigación}

Se trata de una investigación de tipo experimental ya que se espera caracterizar el objeto en estudio señalando sus características y propiedades; así mismo, es de tipo comparativo puesto que se establecerá más de un de tratamiento en la investigación y se compararán entre sí, las respuestas a los diferentes tratamientos.

\section{B. Población y muestra}

Se tiene acceso a los bananos de rechazo del campo experimental de Augura ubicado en el km 1 vía Apartadó - Carepa, Tulenapa. Se toman muestras representativas del material de desecho. 


\section{Diseño del procedimiento}

Una vez realizadas las pruebas de campo y analizadas las consideraciones que inciden en el proceso, se estandariza bajo los siguientes parámetros [19]; descripción del proceso, que comprende la relación de las operaciones y procesos unitarios, con el fin de desarrollar con ello la elaboración de los diagramas de flujo; la relación de equipos y materia prima, los cronogramas de operaciones (etapas y procesos), equipos, mano de obra y servicios industriales para la representación gráfica de las diversas etapas; equipos empleados y proceso general, en función del tiempo transcurrido; cronograma de variables o representación gráfica de las variables que se cambian durante el proceso, en función del tiempo transcurrido, y hojas de proceso y P\&ID, en la cuales se registran las etapas y operaciones así como las variables y tiempos transcurridos.

1) Materiales del proceso: densímetros de vidrio, colador, pilas de hielo, termómetro de vidrio, plancha de calentamiento IKA RH-KT/C, equipo de destilación de vidrio de $2 \mathrm{~L}$, beakers de 1000 $\mathrm{ml}$, probetas de $50 \mathrm{ml}$, balanza analítica Sauter SM 1600, balanza analítica Precisa 240A, pHmetro Metrohm 781, biorreactores.

2) Materias primas: banano de rechazo, levadura Sacharomuces cerevisae, azúcar y agua potable.

\section{Resultados}

Se realizaron 4 pruebas de campo con diferentes grados brix; el desarrollo analítico y experimental se efectuó en las instalaciones del Laboratorio de Aguas de Corpouraba acreditado por el Ideam desde 2006.

\section{A. Descripción del proceso (campo)}

1) Muestreo: el banano de rechazo se tomó de fincas ubicadas en Urabá-Antioquia. La recolección se realizó manualmente; esta es simple e involucra equipamiento mínimo. Se tuvo en cuenta el mejor tipo de banano de descarte disponible para el muestreo.

2) Preparación de la muestra: previamente se dejó madurar la muestra sin que se llegara a descomponer; se adicionó agua potable para obtener un jugo; se estableció el volumen de trabajo; se agregó la levadura; se añadió azúcar hasta llegar al grado brix de interés, midiéndolo con un densímetro de vidrio y homogenizando con una licuadora; se reguló el pH con ácido orto-fosfórico $85 \%$ hasta el intervalo $4.5-5$ y se llevó al respectivo biorreactor.

3) Fermentación: la hidrólisis enzimática de la celulosa se llevó a cabo con enzimas celulasas, catalizadores altamente específicos y bajo condiciones suaves (temperatura entre $20^{\circ} \mathrm{C}$ y $24^{\circ} \mathrm{C}$, proceso anaerobio). Esta etapa tiene una duración de 10 días, en los cuales el control de la temperatura debe ser estricto (para ese fin se utilizaron pilas de hielo y termómetro de vidrio).

4) Descube y destilación: pasado el tiempo, el fermentado se dejó dos días en una nevera a $4^{\circ} \mathrm{C}$; luego se coló para separar sólidos; al líquido se le midió pH y grados brix nuevamente; en el proceso de destilación, se reguló la temperatura del destilado a $98^{\circ} \mathrm{C}$ y se recogió en un recipiente dentro de un baño maría a $4^{\circ} \mathrm{C}$ para evitar pérdidas. Esta etapa duró 4 horas.

5) Identificación del producto: luego de recoger el destilado, se le midió el volumen, el pH y la densidad; de esta manera, se calculó el \% de bioetanol obtenido. 
TABLA I

Balance de Materiales (Campo).

\begin{tabular}{|c|c|c|c|c|c|}
\hline ENSAYO 1 & & & ENSAYO 2 & & \\
\hline Entrada & Unidades & Cantidad & Entrada & Unidades & Cantidad \\
\hline Volumen & Litro & 0.925 & Volumen & Litro & 0.860 \\
\hline Banano & gramos & 171.53 & Banano & gramos & 135.28 \\
\hline Levadura & gramos & 1.063 & Levadura & gramos & 1.078 \\
\hline Grado Brix & ${ }^{\circ} \mathrm{Bx}$ & 14.91 & Grado Brix & ${ }^{\circ} \mathrm{Bx}$ & 24.23 \\
\hline Salida & Unidades & Cantidad & Salida & Unidades & Cantidad \\
\hline Volumen & Litro & 0.146 & Volumen & Litro & 0.067 \\
\hline Bioetanol & $\%(\mathrm{P})$ & 8 & Bioetanol & $\%(P)$ & 21 \\
\hline ENSAYO 3 & & & ENSAYO 4 & & \\
\hline Entrada & Unidades & Cantidad & Entrada & Unidades & Cantidad \\
\hline Volumen & Litro & 0.660 & Volumen & Litro & 0.900 \\
\hline Banano & gramos & 195.00 & Banano & gramos & 128.58 \\
\hline Levadura & gramos & 1.028 & Levadura & gramos & 1.031 \\
\hline Grado Brix & ${ }^{\circ} \mathrm{Bx}$ & 17.79 & Grado Brix & ${ }^{\circ} \mathrm{Bx}$ & 20.39 \\
\hline Salida & Unidades & Cantidad & Salida & Unidades & Cantidad \\
\hline Volumen & Litro & 0.042 & Volumen & Litro & 0.105 \\
\hline Bioetanol & $\%(P)$ & 12 & Bioetanol & $\%(P)$ & 16 \\
\hline
\end{tabular}


TABLA II

Cronograma de Variables (campo)

\begin{tabular}{|c|c|c|c|}
\hline \multicolumn{4}{|c|}{ CRONOGRAMA DE VARIABLES } \\
\hline VARIABLES DE ENTRADA & Unidades & Cantidad & Tiempo \\
\hline Masa ensayo 1 & gramos & 171.53 & 15 minutos \\
\hline Masa ensayo 2 & gramos & 135.28 & 15 minutos \\
\hline Masa ensayo 3 & gramos & 195.00 & 15 minutos \\
\hline Masa ensayo 4 & gramos & 128.58 & 15 minutos \\
\hline${ }^{\circ}$ Bx Ensayo 1 & ${ }^{\circ} \mathrm{Bx}$ & 14.91 & 30 minutos \\
\hline${ }^{\circ}$ Bx Ensayo 2 & ${ }^{\circ} \mathrm{Bx}$ & 24.23 & 30 minutos \\
\hline${ }^{\circ}$ Bx Ensayo 3 & ${ }^{\circ} \mathrm{Bx}$ & 17.79 & 30 minutos \\
\hline${ }^{\circ}$ Bx Ensayo 4 & ${ }^{\circ} \mathrm{Bx}$ & 20.39 & 30 minutos \\
\hline Volumen a destilar ensayo 1 & Litros & 0.925 & N.A \\
\hline Volumen a destilar ensayo 2 & Litros & 0.860 & N.A \\
\hline Volumen a destilar ensayo 3 & Litros & 0.660 & N.A \\
\hline Volumen a destilar ensayo 4 & Litros & 0.900 & N.A \\
\hline VARIABLES DE SALIDA & Unidades & Cantidad & Tiempo \\
\hline Volumen destilado ensayo 1 & Litros & 0.146 & 4 Horas \\
\hline Volumen destilado ensayo 2 & Litros & 0.067 & 4 Horas \\
\hline Volumen destilado ensayo 3 & Litros & 0.042 & 4 Horas \\
\hline Volumen destilado ensayo 4 & Litros & 0.105 & 4 Horas \\
\hline$\%(P)$ Bioetanol ensayo 1 & $\%(P)$ & 8 & 4 Horas \\
\hline$\%(P)$ Bioetanol ensayo 2 & $\%(P)$ & 21 & 4 Horas \\
\hline$\%(P)$ Bioetanol ensayo 3 & $\%(P)$ & 12 & 4 Horas \\
\hline$\%(\mathrm{P})$ Bioetanol ensayo 4 & $\%(P)$ & 16 & 4 Horas \\
\hline
\end{tabular}

6) Variables críticas: para realizar los análisis de varianza, se observó que las respuestas significativas se debían principalmente al valor de los Brix y al volumen por destilar. 
TABLA III

VARIABLES CRÍTICAS

\begin{tabular}{|c|c|c|c|}
\hline X1 & X2 & Y1 & Y2 \\
\hline${ }^{\mathbf{O}}$ Brix & Volumen a Destilar & Volumen Destilado & \%(P) Bioetanol \\
\hline 14.91 & 0.925 & 0.146 & 8 \\
\hline 24.23 & 0.860 & 0.067 & 21 \\
\hline 17.79 & 0.660 & 0.042 & 12 \\
\hline 20.39 & 0.900 & 0.105 & 16 \\
\hline
\end{tabular}

Para el análisis estadístico del \%(P) Bioetanol (Y2), utilizando la herramienta de Excel "análisis de datos" en regresión, se obtuvieron los siguientes resultados:

TABLA IV

Estadísticas de LA REgResión Y2

\begin{tabular}{|l|c|}
\hline \multicolumn{2}{|c|}{ Estadísticas de la regresión } \\
\hline Coeficiente de correlación múltiple & 0,99956657 \\
\hline Coeficiente de determinación $\mathrm{R}^{\wedge} 2$ & 0,999133327 \\
\hline $\mathrm{R}^{\wedge} 2$ ajustado & 0,997399982 \\
\hline Error típico & 0,283520538 \\
\hline Observaciones & 4 \\
\hline
\end{tabular}

7) Criterio: se plantearon dos hipótesis bajo el criterio de la "pertinencia", ya que aclaran la necesidad de abordar del problema.

8) Hi1: los ${ }^{\circ}$ Brix aportan significativamente al \%(P) de bioetanol obtenido.

9) Hi2: el volumen a destilar aporta significativamente al \%(P) de bioetanol obtenido.

TABLA V

ANOVA Y2

\begin{tabular}{|c|c|c|c|c|c|c|c|c|c|}
\hline \multicolumn{2}{|c|}{ ANÁLISIS DE VARIANZA } & $\begin{array}{c}\text { Grados de } \\
\text { libertad }\end{array}$ & \multicolumn{2}{|c|}{$\begin{array}{l}\text { Suma de } \\
\text { cuadrados }\end{array}$} & \multicolumn{2}{|c|}{$\begin{array}{l}\text { Promedio de los } \\
\text { cuadrados }\end{array}$} & $\mathrm{F}$ & \multicolumn{2}{|c|}{ Valor crítico de $F$} \\
\hline \multirow{4}{*}{$\begin{array}{l}\text { Regresión } \\
\text { Residuos } \\
\text { Total }\end{array}$} & & 2 & \multicolumn{2}{|c|}{92,6696161} & \multicolumn{2}{|c|}{46,33480805} & \multirow[t]{3}{*}{576,419042} & \multicolumn{2}{|c|}{0,029439306} \\
\hline & & 1 & \multicolumn{2}{|c|}{0,080383895} & \multicolumn{2}{|c|}{0,080383895} & & & \\
\hline & & 3 & 92 & & & & & & \\
\hline & Coeficientes & $\begin{array}{l}\text { Error } \\
\text { típico }\end{array}$ & $\underset{t}{\text { Estadístico }}$ & Prob & abilidad & $\begin{array}{c}\text { Inferior } \\
95 \%\end{array}$ & $\begin{array}{l}\text { Superior } \\
95 \%\end{array}$ & $\begin{array}{l}\text { Inferior } \\
95,0 \%\end{array}$ & $\begin{array}{l}\text { Superior } \\
95,0 \%\end{array}$ \\
\hline Intercepción & -13.347 & 1.368 & -9.754 & & 065 & -30.734 & 4.038 & -30.734 & 4.038 \\
\hline${ }^{\circ}$ Brix & 1.402 & 0.041 & 33.899 & & 018 & -0.876 & 1.928 & -0.876 & 1.928 \\
\hline $\begin{array}{l}\text { Volumen a } \\
\text { Destilar }\end{array}$ & 0.579 & 1.359 & 0.426 & & 743 & -16.696 & 17.855 & -16.696 & 17.855 \\
\hline
\end{tabular}


TABLA VI

ANÁlisis de Residuales Y2

\begin{tabular}{|c|c|c|c|c|c|}
\hline \multicolumn{3}{|c|}{ Análisis de los residuales } & \multicolumn{2}{c|}{$\begin{array}{c}\text { Resultados de datos } \\
\text { de probabilidad }\end{array}$} \\
\hline Observación & $\begin{array}{c}\text { Pronóstico Volumen } \\
\text { Destilado }\end{array}$ & Residuos & Residuos estándares & Percentil & Volumen Destilado \\
\hline 1 & 0.146 & -0.000 & -0.621 & 12.5 & 8 \\
\hline 2 & 0.067 & -0.000 & -0.836 & 37.5 & 12 \\
\hline 3 & 0.042 & 0.000 & 0.075 & 62.5 & 16 \\
\hline 4 & 0.104 & 0.001 & 1.382 & 87.5 & 21 \\
\hline
\end{tabular}

Debido a que F>0.029 y el valor del estadístico t correspondiente a los Brix son los únicos mayores que al de la probabilidad $(33,899>0,0187)$, se considera que el nivel de los resultados son estadísticamente significativos y representativos; además, los valores de los coeficientes sugieren que los ${ }^{\circ}$ Brix influyen casi 3 veces más en la obtención de bioetanol que el volumen por destilar, por lo tanto, se acepta la hipótesis Hi1.

Para el análisis estadístico del volumen destilado (Y1), utilizando la herramienta de Excel "análisis de datos" en regresión, se obtuvieron los siguientes resultados:

TABLA VII

EstadísticAs DE LA REGRESIÓN Y1

\begin{tabular}{|l|c|}
\hline \multicolumn{2}{|c|}{ Estadísticas de la regresión } \\
\hline Coeficiente de correlación múltiple & 0,999931425 \\
\hline Coeficiente de determinación $\mathrm{R}^{\wedge} 2$ & 0,999862855 \\
\hline $\mathrm{R}^{\wedge} 2$ ajustado & 0,999588566 \\
\hline Error típico & 0,000921669 \\
\hline Observaciones & 4 \\
\hline
\end{tabular}

10) Criterio: se plantearon dos hipótesis bajo el criterio de la "pertinencia" ya que aclaran la necesidad de abordar del problema.

11) Hi1: los ${ }^{\circ}$ Brix aportan significativamente al volumen destilado obtenido.

12) Hi2: el volumen por destilar aporta significativamente al volumen destilado obtenido. 
TABLA VIII

ANOVA Y1

\begin{tabular}{|c|c|c|c|c|c|c|c|c|c|c|c|}
\hline \multicolumn{12}{|c|}{ ANÁLISIS DE VARIANZA } \\
\hline & & & \multicolumn{2}{|c|}{$\begin{array}{l}\text { Grados de } \\
\text { libertad }\end{array}$} & \multicolumn{2}{|c|}{$\begin{array}{l}\text { Suma de } \\
\text { cuadrados }\end{array}$} & \multicolumn{2}{|c|}{$\begin{array}{l}\text { Promedio de los } \\
\text { cuadrados }\end{array}$} & os & \multicolumn{2}{|c|}{ Valor crítico de F } \\
\hline \multirow{4}{*}{$\begin{array}{l}\text { Regresión } \\
\text { Residuos } \\
\text { Total }\end{array}$} & & & \multicolumn{2}{|l|}{2} & \multicolumn{2}{|c|}{0,006193151} & \multicolumn{2}{|c|}{0,003096575} & \multirow[t]{3}{*}{3645,286} & \multicolumn{2}{|c|}{0,011710876} \\
\hline & & & \multicolumn{2}{|l|}{1} & \multicolumn{2}{|c|}{ 8,49474E-07 } & \multicolumn{2}{|c|}{ 8,49474E-07 } & & & \\
\hline & & & \multicolumn{2}{|l|}{3} & \multicolumn{2}{|c|}{0,006194} & & & & & \\
\hline & \multicolumn{2}{|c|}{ Coeficientes } & $\begin{array}{l}\text { Error } \\
\text { típico }\end{array}$ & \multicolumn{2}{|c|}{$\underset{\mathrm{t}}{\text { Estadístico }}$} & \multicolumn{2}{|c|}{ Probabilidad } & $\begin{array}{l}\text { Inferior } \\
95 \%\end{array}$ & $\underset{95 \%}{\text { Superior }}$ & $\begin{array}{l}\text { Inferior } \\
95,0 \%\end{array}$ & $\begin{array}{c}\text { Superior } \\
95,0 \%\end{array}$ \\
\hline Intercepción & \multicolumn{2}{|c|}{-0.064} & 0.004 & \multicolumn{2}{|c|}{-14.288} & \multicolumn{2}{|c|}{0.044} & -0.120 & -0.007 & -0.120 & -0.007 \\
\hline${ }^{\circ}$ Brix & \multicolumn{2}{|c|}{-0.006} & 0.000 & \multicolumn{2}{|c|}{-45.991} & \multicolumn{2}{|c|}{0.014} & -0.008 & -0.004 & -0.008 & -0.004 \\
\hline $\begin{array}{l}\text { Volumen a } \\
\text { Destilar }\end{array}$ & \multicolumn{2}{|c|}{0.327} & 0.004 & \multicolumn{2}{|c|}{73.898} & \multicolumn{2}{|c|}{0.009} & 0.270 & 0.383 & 0.270 & 0.383 \\
\hline \multicolumn{12}{|c|}{$\begin{array}{c}\text { TABLA IX } \\
\text { ANÁLISIS DE RESIDUALES Y1. }\end{array}$} \\
\hline \multicolumn{2}{|c|}{$\begin{array}{l}\text { Análisis de los } \\
\text { residuales }\end{array}$} & & & & & & & & \multicolumn{3}{|c|}{$\begin{array}{l}\text { Resultados de datos } \\
\text { de probabilidad }\end{array}$} \\
\hline Observacic & & $\begin{array}{r}\text { Pronóst } \\
\text { De }\end{array}$ & $\begin{array}{l}\text { ico Volum } \\
\text { stilado }\end{array}$ & en & Residuo & & $\begin{array}{l}\text { Residur } \\
\text { stánda }\end{array}$ & & Percentil & Volumen & Jestilado \\
\hline 1 & & & 0.146 & & -0.000 & & -0.62 & & 12.5 & 0.0 & \\
\hline 2 & & & 0.067 & & -0.000 & & -0.836 & & 37.5 & 0.0 & \\
\hline 3 & & & 0.042 & & 0.000 & & 0.075 & & 62.5 & 0.1 & \\
\hline 4 & & & 0.104 & & 0.001 & & 1.382 & & 87.5 & 0.1 & 46 \\
\hline
\end{tabular}

Debido a que F>0.0117 y el valor del estadístico $\mathrm{t}$ correspondiente al volumen por destilar son los únicos mayores que al de la probabilidad $(73,89>0,0086)$, se considera que el nivel de los resultados son estadísticamente significativos y representativos; además, los valores de los coeficientes sugieren que el volumen por destilar es el único que influye en el volumen destilado, ya que el de los ${ }^{\circ}$ Brix es negativo; por lo tanto, se acepta la hipótesis $\mathrm{Hi2}$.

\section{B. Balance parcial de materiales para la ob- tención de bioetanol}

Se realiza un balance global de los diferentes procesos; aunque se incluye el ensayo 3 , este no participa en los cálculos debido a que tiene diferente base de trabajo; se calculan promedios y se realiza una simulación a mayor escala. 
TABLA X

Balance Parcial Global

\begin{tabular}{|c|c|c|c|c|c|c|c|c|}
\hline ENSAYOS & & PRIMERO & SEGUNDO & TERCERO & CUARTO & \multicolumn{2}{|c|}{ PROMEDIO } & SIMULACIÓN \\
\hline Volumen base de trabajo & & $1 \mathrm{~L}$ & $1 \mathrm{~L}$ & $0,6 \mathrm{~L}$ & $1 \mathrm{~L}$ & & & $1000 \mathrm{~L}$ \\
\hline \multicolumn{9}{|l|}{ RECIBO DE MATERIA PRIMA } \\
\hline & Simbolo & Kilos & Kilos & Kilos & Kilos & kilos & PORCENT. & kilos \\
\hline \multicolumn{9}{|l|}{ ENTRADAS } \\
\hline Banano verde & $\mathrm{Bv}$ & 0.236 & 0.238 & 0.240 & 0.234 & 0.236 & $100.00 \%$ & 236.27 \\
\hline TOTAL ENTRADAS & & 0.236 & 0.238 & 0.240 & 0.234 & 0.236 & $100.00 \%$ & 236.27 \\
\hline \multicolumn{9}{|l|}{ SALIDAS } \\
\hline Banano Madurado & $\mathrm{Bm}$ & 0.172 & 0.135 & 0.195 & 0.129 & 0.145 & $61.43 \%$ & 145.13 \\
\hline Rechazo (Cáscara) & $\mathrm{R}$ & 0.065 & 0.103 & 0.045 & 0.106 & 0.091 & $38.57 \%$ & 91.14 \\
\hline TOTAL SALIDAS & & 0.236 & 0.238 & 0.240 & 0.234 & 0.236 & $100 \%$ & 236.27 \\
\hline \multicolumn{9}{|l|}{ ADECUACIÓN BANANO } \\
\hline \multicolumn{9}{|l|}{ ENTRADAS } \\
\hline Banano madurado & $\mathrm{Bm}$ & 0.172 & 0.135 & 0.195 & 0.129 & 0.145 & $13.40 \%$ & 145.13 \\
\hline Agua & W & 0.800 & 0.800 & 0.500 & 0.800 & 0.800 & $73.85 \%$ & 800.00 \\
\hline Azúcar & $A$ & 0.093 & 0.164 & 0.079 & 0.156 & 0.138 & $12.71 \%$ & 137.71 \\
\hline Acido Ortofosfórico 85\% & H3P04 & 0.001 & 0.001 & 0.001 & 0.001 & 0.001 & $0.05 \%$ & 0.50 \\
\hline TOTAL ENTRADAS & & 1.065 & 1.100 & 0.774 & 1.085 & 1.083 & $100.00 \%$ & 1083.34 \\
\hline \multicolumn{9}{|l|}{ SALIDAS } \\
\hline Mosto & Mt & 1.054 & 1.089 & 0.766 & 1.074 & 1.073 & $99.00 \%$ & 1072.51 \\
\hline Merma & Me & 0.011 & 0.011 & 0.008 & 0.011 & 0.011 & $1.00 \%$ & 10.83 \\
\hline TOTAL SALIDAS & & 1.065 & 1.100 & 0.774 & 1.085 & 1.083 & $100.00 \%$ & 1083.34 \\
\hline \multicolumn{9}{|l|}{ FERMENTACIÓN } \\
\hline \multicolumn{9}{|l|}{ ENTRADAS } \\
\hline Mosto & Mt & 1.054 & 1.089 & 0.766 & 1.074 & 1.073 & $99.90 \%$ & 1072.51 \\
\hline Levadura & Le & 0.001 & 0.001 & 0.001 & 0.001 & 0.001 & $0.10 \%$ & 1.06 \\
\hline TOTAL ENTRADAS & & 1.055 & 1.090 & 0.767 & 1.075 & 1.074 & $100.00 \%$ & 1073.56 \\
\hline \multicolumn{9}{|l|}{ SALIDAS } \\
\hline Fermentado & $\mathrm{Fm}$ & 0.981 & 1.032 & 0.726 & 1.030 & 1.014 & $94.47 \%$ & 1014.16 \\
\hline Levadura y residuos sólidos & Lrs & 0.005 & 0.005 & 0.005 & 0.005 & 0.005 & $0.49 \%$ & 5.29 \\
\hline Gas carbónico & Gc & 0.070 & 0.053 & 0.036 & 0.040 & 0.054 & $5.04 \%$ & 54.11 \\
\hline TOTAL SALIDAS & & 1.055 & 1.090 & 0.767 & 1.075 & 1.074 & $100.00 \%$ & 1073.56 \\
\hline \multicolumn{9}{|l|}{ DESTILACIÓN } \\
\hline \multicolumn{9}{|l|}{ ENTRADAS } \\
\hline Fermentado & $\mathrm{Fm}$ & 0.981 & 1.032 & 0.726 & 1.030 & 1.014 & $100 \%$ & 1014.16 \\
\hline TOTAL ENTRADAS & & 0.981 & 1.032 & 0.726 & 1.030 & 1.014 & $100 \%$ & 1014.16 \\
\hline \multicolumn{9}{|l|}{ SALIDAS } \\
\hline Destilado (Agua + Alcohol) & Dt & 0.145 & 0.065 & 0.041 & 0.102 & 0.104 & $9.30 \%$ & 94.28 \\
\hline Alcohol & $\mathrm{Al}$ & 0.012 & 0.014 & 0.005 & 0.016 & 0.014 & $1.24 \%$ & 12.59 \\
\hline Agua & W & 0.133 & 0.051 & 0.036 & 0.086 & 0.090 & $8.06 \%$ & 81.69 \\
\hline Vinazas & Vz & 0.836 & 0.967 & 0.685 & 0.928 & 0.910 & $81.41 \%$ & 825.61 \\
\hline TOTAL SALIDAS & & 0.981 & 1.032 & 0.726 & 1.030 & 1.118 & $100.00 \%$ & 1014.16 \\
\hline$\%$ Alcohol Obtenido (\%P) & & 8 & 21 & 12 & 16 & 14.252 & & 15.03 \\
\hline Volumen Destilado (L) & & 0.147 & 0.067 & 0.042 & 0.105 & 0.106 & & 106.3 \\
\hline Densidad Obtenida Destilado & & 0.985 & 0.965 & 0.980 & 0.975 & 0.975 & & 0.975 \\
\hline Densidad Obtenida Inicial & & 1.065 & 1.100 & 1.075 & 1.085 & 1.083 & & 1.083 \\
\hline
\end{tabular}




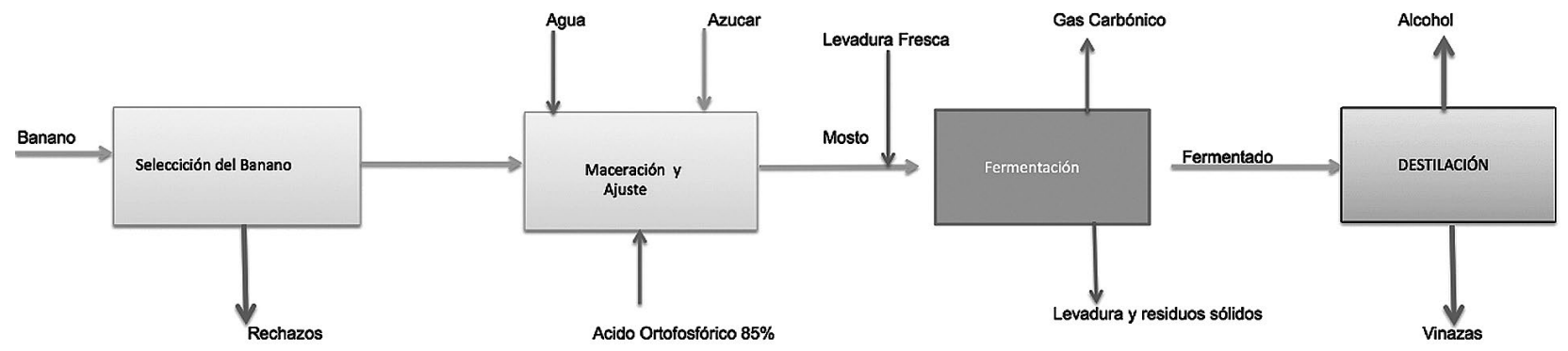

Fig. 1 Diagrama elemental del proceso

TABLA XI

Cronograma Global del Proceso.

\begin{tabular}{|c|c|c|c|c|c|c|c|c|c|c|c|c|c|c|c|c|}
\hline ETAPA & OPERACION & HORA & 8,0 & 8,5 & 9,0 & 9,5 & \begin{tabular}{l|l}
10,0 & 10,5 \\
\end{tabular} & 11,0 & 11,5 & 12,0 & 12,5 & 13,01 & \begin{tabular}{l|l}
13,5 & 1 \\
\end{tabular} & \begin{tabular}{l|l}
4,0 & 14 \\
\end{tabular} & \begin{tabular}{|l|l|}
4,5 & 15,0 \\
\end{tabular} & \begin{tabular}{|l|l|}
15,5 & 16,0 \\
\end{tabular} \\
\hline \multirow{3}{*}{ Adecuación de materias } & Recepciòn (transporte) & & & & & & & & & & & & & & & \\
\hline & Selección (separación) & & & & & & & & & & & & & & & \\
\hline & Aseo equipos & & & & & & & & & & & & & & & \\
\hline \multirow{5}{*}{ Maceracion y ajustes } & Molienda (reducción de tamaño) & & & & & & & & & & & & & & & \\
\hline & Adición de agua & & & & & & & & & & & & & & & \\
\hline & Adición de azucar (mezcla) & & & & & & & & & & & & & & & \\
\hline & Bombeo a fermentación & & & & & & & & & & & & & & & \\
\hline & Aseo equipos & & & & & & & & & & & & & & & \\
\hline
\end{tabular}

PARA QUINCE DIAS DE TRABAJO

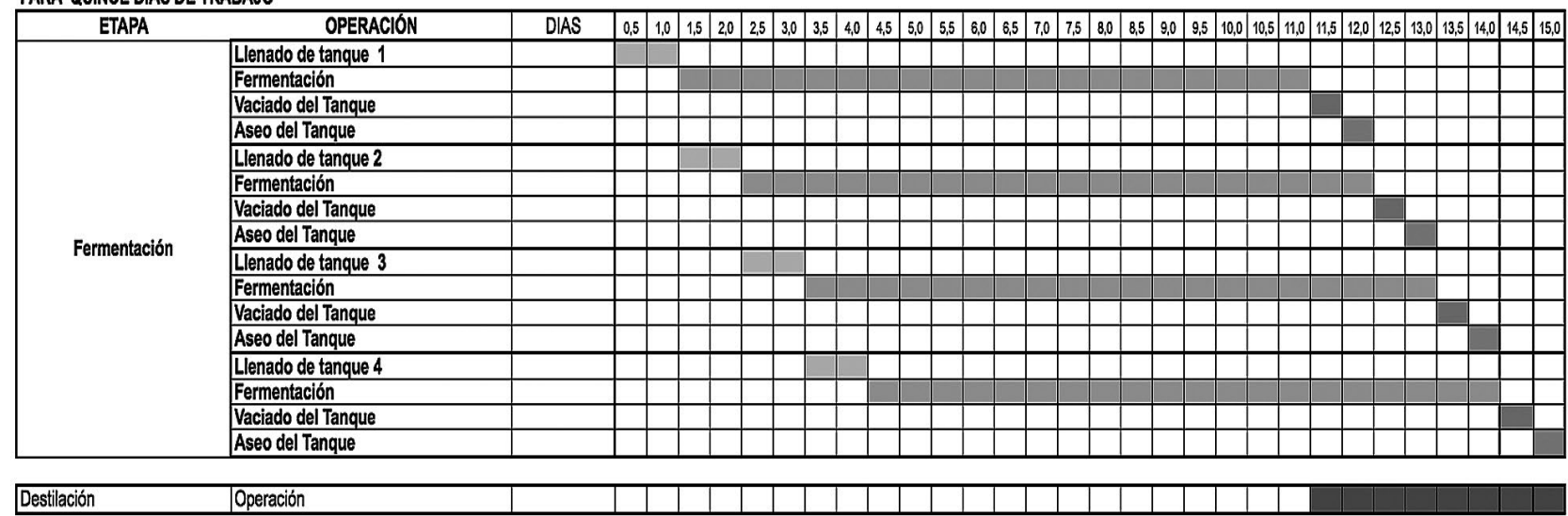


TABLA XII

HoJa de Proceso

\begin{tabular}{|c|c|c|c|c|c|}
\hline \multicolumn{6}{|c|}{ PROCESO DE OBTENCIÓN DE BIOETANOL DEL RECHAZO DE BANANO } \\
\hline \multicolumn{2}{|l|}{ Hoja de proceso $\mathrm{N}^{0}$ : } & & \multicolumn{3}{|c|}{ Fecha:________-__-__-_-___-_ } \\
\hline & \multicolumn{2}{|c|}{ Tiempo (Días) } & & & \\
\hline $\begin{array}{l}\text { Alistamiento Materias } \\
\text { Primas }\end{array}$ & & Acumulado & De & Hasta & Flujo \\
\hline Selección/Muestreo & 0.5 & 0.5 & & & \\
\hline Recepción & 0.5 & 1 & & & \\
\hline $\begin{array}{l}\text { Banano rechazo verde a } \\
\text { maduración }\end{array}$ & 4 & 5 & & & \\
\hline \multicolumn{6}{|l|}{ Tiempo total } \\
\hline & \multicolumn{2}{|c|}{ Tiempo (Días) } & & & \\
\hline Preparación de la muestra & & Acumulado & De & Hasta & Flujo \\
\hline Grados Brix y licuado & 0.5 & 0.5 & & & \\
\hline pH regulado de 4,5 - 5 & 0.5 & 1 & & & \\
\hline \multicolumn{6}{|l|}{ Tiempo total } \\
\hline & \multicolumn{2}{|c|}{ Tiempo (Días) } & & & \\
\hline Fermentación anaerobia & & Acumulado & De & Hasta & Flujo \\
\hline Transporte al fermentador & 0.1 & 0.1 & & & \\
\hline Adición de levadura & 0.1 & 0.2 & & & \\
\hline $\begin{array}{l}\text { Control de temperatura } \\
20^{\circ} \mathrm{C}-24^{\circ} \mathrm{C}\end{array}$ & 4.8 & 5 & & & \\
\hline \multicolumn{6}{|l|}{ Tiempo total } \\
\hline & \multicolumn{2}{|c|}{ Tiempo (Días) } & & & \\
\hline Destilación & & Acumulado & De & Hasta & Flujo \\
\hline Transporte al destilador & 0.1 & 0.1 & & & \\
\hline Destilación controlada & 0.9 & 1 & & & \\
\hline \multicolumn{6}{|l|}{ Tiempo total } \\
\hline Materias Primas & $\mathrm{Kg}$ & & Turno & Operario & \\
\hline \multicolumn{6}{|l|}{ Banano de rechazo } \\
\hline \multicolumn{6}{|l|}{ Levadura } \\
\hline \multicolumn{6}{|l|}{ Azucar } \\
\hline Agua & & & & & \\
\hline Total & & & & & \\
\hline
\end{tabular}

\section{Conclusiones}

Tras la realización del análisis para una posible solución al problema planteado, con vistas al logro de una mayor optimización (diseño de una planta de obtención de bioetanol), es posible concluir que:
Los procesos unitarios necesarios para la obtención del bioetanol a partir del banano de rechazo se consultaron y aplicaron de forma controlada logrando una óptima fermentación. 
Las pruebas de campo se desarrollaron satisfactoriamente capturando una muestra representativa del problema por tratar; además, los análisis y ensayos experimentales se realizaron en un laboratorio acreditado por el Ideam, bajo excelentes condiciones instrumentales y ambientales avalando así el ejercicio analítico.

El jugo de banano presenta un alto potencial de uso como sustrato en los procesos fermentativos para la producción de alcohol. Con este sustrato se lograron altos rendimientos y una conversión de más del $80 \%$ con respecto a los valores teóricos reportados en tablas de conversión Brix, lo que lo convierte en un sustrato promisorio para las fermentaciones alcohólicas.

La estandarización se desarrolló de manera satisfactoria utilizando los datos arrojados por el trabajo de campo; se logró la compilación necesaria que se había establecido como requisito para un futuro diseño de una planta de obtención de bioetanol a partir del banano de rechazo.

Existen en la actualidad más de 300 fincas bananeras en la región, las cuales realizan en promedio embarques de 5 días a la semana, y aunque parte de los bananos de rechazo generados se destinan para el consumo interno, esto no sería limitación alguna para la captura de materia prima.

Como resultado de la investigación presentada, es posible concluir que el aprovechamiento del banano de rechazo de la región de Urabá como materia prima en la obtención de bioetanol no solo aporta una solución más al problema de residuos agroindustriales sino que además es una potencial fuente de recursos bioenergéticos, oportunidad laboral y de inversión regional ya que esta biotecnología se viene proyectando en el mundo con resultados muy satisfactorios.

\section{ReCOMENDACIONES}

Una vez concluida la tesis, se considera interesante investigar sobre otros aspectos relacionados con el mecanismo utilizado de aprovechamiento del banano y su problemática residual. Se propone, entonces:

Realizar otro trabajo de investigación similar utilizando una levadura termo resistente o al menos que no necesite un control de temperatura tan estricto ya que Urabá es una zona cálida tropical; esto podría facilitar el proceso de fermentación.

Tomando como referencia la problemática residual se podría realizar una investigación en el aprovechamiento de dicho residuo fibroso y la calidad de celulosa que se puede obtener.

Otro subproducto del cultivo de banano es el bagazo, el cual es rico en material celulósico.

\section{RefERENCIAS}

[1] I.D.Uyazán, J.L, Gil, G. Aguilar, L.A. Rodrìguez, Caicedo. "Deshidratación del etanol", Revista ingenieros e investigación no. 56,pp. 49-59, Dic. 2004.

[2] Alvear M. R., Castillo C. R., Henao D. L., Marimón W., Tejada C. N., Tejeda L. P., Villabona A. (2009). Estudio de la hidrólisis ácida de cáscaras de naranja citrus síntesis para la obtención de etanol. Memorias del IV Simposio de Química Aplicada - SIQUIA 2009 p. 1-8.

[3] L.P. Tejada, C. Tejada, A. Villabona, M. R. Alvear Mario C.R. Castillo Carlos, D.L. Henao D, Marimón Wilfredo, N. Madariaga, A. Tarón, "Producción de bioetanol a partir de la fermentación alcohólica de jarabes glucosados derivados de cáscaras de naranja y piña", Revista Educación en Ingeniería no.. 10 pp. 120-125, 2010.

[4] A.M. Afanador, "El banano verde de rechazo en la producción de alcohol carburante", Revista EIA, ISSN 1794-1237, Escuela de Ingeniería de Antioquia, Medellín. NO. 3 pP. 51-68,M 2005.

[5] A.M. Zapata (.2010) "Producción en continuo de etanol a partir de banano de rechazo (cáscara y pulpa) empleando células inmovilizadas." Instituto de QuímicaUniversidad de Antioquia. Revista Tumbaga 5 49-60. 
[6] Desarrollo regional en Uraba, Colombia, (2011). La materia orgánica es factor limitante, disponible en http://www.bio-tec.net/archivos/News /2011/ MAYO/ PC30-C78\%20(E-news\%20Urab\%C3\%A1\% 20art\%C3\%ADculo)\%20Mayo\%206-11.pdf visitado el 02/05/2014.

[7] T. Bastidas, D. Ibarra, S. Serrano S., D. Salgado D., V. Benítez, (2010), Aislamiento, selección y preservacion de cepas para la obtención de bioetanol a partir de residuos de banano, disponible en http:// es.scribd.com/doc/34026364/PROYECTO-bananoNMB visitado el 02/05/2014.

[8] G.T. Austin, G. T. Shreve's Chemical Process Industries: Chapter 31. Fermentation industries. 5th edition. McGraw-Hill, 1984. p. 581-591.

[9] H. Velásquez, Análisis energético y exergético del proceso de obtención de etanol a partir de la fruta del banano. Grupo de Investigación en Bioprocesos, Facultad de Minas Universidad Nacional de Colombia: Medellín, Colombia. 2010.

[10] K. Cruz, C. Suárez, A. Gómez, B. Otálvaro, B., Optimización de las condiciones de operación de la hidrólisis enzimática sobre el material lignocelulósico de la planta de banano y su fruto. Universidad $\mathrm{Na}$ cional de Colombia, grupo de bioprocesos y flujos reactivos. Medellín, Colombia, 2004.

[11] J. García, C. y J. García L. Biocarburantes líquidos: Biodiésel y Bioetanol. Informe de Vigilancia Tecnológica. Fundacion para el conocimiento Madrid CEIM, 2006.

[12] L.C. Meher, S. Vidga, S.N. Naik, "Technical aspects of biodesel production by transesterification - a review". Renewable \& Sustainable Energy Reviews, vol 10, (3): pp. 248-268, 2006.
[13] J. Monsalve, V. Medina, A. Ruiz, Producción de etanol a partir de la cáscara de banano y de almidón de yuca. Universidad Nacional de Colombia. Grupo de Bioprocesos-grupo de combustibles alternativos, Medellín, Colombia, 2006.

[14] [G. Vicente, M. Martínez y J. Aracil, "Biodiésel: una alternativa real al gasóleo mineral". Ingeniería Química 377 (3), pp. 135-145, 2001.

[15] F.R. Peralta, J. Bonnabel, (2010). Etanol una opción viable de combustible, disponible en http:// www.tlalpan.uvmnet.edu/oiid/download/ Etanol\%20 como\%20combustible_04_ING_IMI_PIT_E.pdf visitado el 02/05/2014.

[16] N. Montes y L. Torres, (2004). Hidrólisis enzimática de banano verde de rechazo. Medellín, 101p. Trabajo de grado (Ingeniero Químico). Universidad Nacional de Colombia, Facultad de Minas.

[17] Martínez, A. Lopera, L. (2003). "Evaluación del rendimiento de etanol en una fermentación continua con Zymomonas mobilis". Trabajo de grado (Ingeniero Químico). Universidad Nacional de Colombia, Facultad de Minas, Medellín, 2003.

[18] O. Masera, C. et, al., Potenciales y Viabilidad del Uso de Bioetanol y Biodiesel para el transporte en Mexico. Secretaría de Energía. pp. 151 - 300, 2006.

[19] V. Fonseca, V, Ingeniería de procesos en las industrias de alimentos y bebidas. Módulo UNAD Estandarización de procesos en la industria de alimentos. Universidad Abierta Distancia, Bogotá, 2011. 